\title{
Pretreatment albumin globulin ratio has a superior prognostic value in laryngeal squamous cell carcinoma patients: a comparison study
}

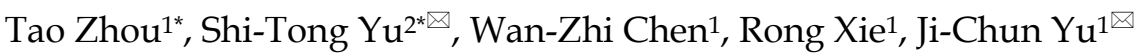 \\ 1. Department of Thyroid and Neck Surgery, The Second Affiliated Hospital of Nanchang University, Nanchang, Jiangxi, China. \\ 2. Department of General Surgery, Nanfang Hospital, Southern Medical University, Guangzhou, Guangdong, China. \\ *These authors contributed equally to this work \\ $\bowtie$ Corresponding authors: Shi-Tong Yu. E-mail: yusht3@mail2.sysu.edu.cn; Ji-Chun Yu. E-mail: yjchns@126.com \\ (c) Ivyspring International Publisher. This is an open access article distributed under the terms of the Creative Commons Attribution (CC BY-NC) license \\ (https://creativecommons.org/licenses/by-nc/4.0/). See http://ivyspring.com/terms for full terms and conditions.
}

Received: 2018.07.27; Accepted: 2018.12.14; Published: 2019.01.01

\begin{abstract}
Background: Many inflammation-based markers have been reported their prognostic significance. Current study was designed to explore the prognostic value of albumin/globulin ratio (AGR), along with other inflammation-based markers, including neutrophil/lymphocyte ratio (NLR), platelet/lymphocyte ratio (PLR) and lymphocyte/monocyte ratio (LMR) in laryngeal squamous cell carcinoma (LSCC) patients.

Method: This study was a retrospective analysis of the data related to 232 newly diagnosed LSCC patients. The potential prognostic factors were evaluated by univariate and multivariate survival analysis. The correlation between AGR and other prognostic factors were analyzed, and the area under the curve (AUC) were compared.

Results: AGR, NLR, PLR and LMR were found to be associated with several aggressive clinicopathological features and poor prognosis. In multivariate analysis, AGR, NLR, PLR, LMR were independent prognostic markers of the shorter OS. However, NLR, PLR, and LMR showed no significance with the shorter DFS. AGR remained an independent prognostic marker for the shorter DFS. Furthermore, AGR was a superior prognosis factor than NLR, PLR, LMR in LSCC patients.

Conclusion: AGR might be a promising marker to better predicting prognosis of LSCC patients. Future studies are warranted to validate our finding.
\end{abstract}

Key words: inflammation-based markers; albumin/globulin ratio; laryngeal squamous cell carcinoma; prognosis

\section{Background}

In 2017, laryngeal squamous cell carcinoma (LSCC) accounts for 13150 newly diagnosed and 3710 cancer-related deaths annually in the United States [1]. In 2015, the number reported in China was 26400 and 14500 respectively [2]. Tumor-Node-Metastasis (TNM) staging system is the most commonly used methodology in evaluating LSCC prognosis. However, there were some inter-individual differences observed in the same TNM stage LSCC patients. One possible reason is the case that the current TNM staging system does not fully explain tumor heterogeneity.

Recent studies have shown that chronic inflammation increasing the risk in many malignancies, including LSCC [3-6]. Neutrophils, as an inflammatory cell, could promote tumor cell growth and invasion by generating cytokines and vascular endothelial growth factors (VEGF) [7-9]. Platelets could assist tumor cells escaped from antitumor immunity and secret VEGF and platelet-derived growth factors (PDGF) [7, 10, 11]. Lymphocytes could stop tumor progression, and reflect the function of patients' immune system [12]. Thus, some inflammation markers, including neutrophil/lymphocyte ratio (NLR), platelet/ lymphocyte ratio (PLR) and lymphocyte/monocyte ratio (LMR), have been established to play a prognostic role in LSCC [13-17]. In previous study, we 
described a novel inflammation-based marker, albumin/globulin ratio (AGR) has a prognostic significance in LSCC [18]. However, the comparison of these markers in LSCC patients have not been investigated.

Therefore, the aim of current study was to explore and compare the prognostic value of different inflammation-based markers in an independent LSCC patient cohort, including AGR, NLR, PLR and LMR.

\section{Material and methods}

\section{Patients}

Three hundred and sixty-five patients with firstly diagnosed LSCC were retrospectively enrolled from January 2008 to December 2013. All surgeries were performed by the same experienced surgeon (Ji-Chun $\mathrm{Yu}$ ) at the First/Second Affiliated Hospital of Nanchang University, China. The exclusion criteria were as following: (1) received any anti-cancer therapy previously (including radiotherapy/ induction chemotherapy), $\mathrm{n}=56$; (2) a history of previous/synchronous malignant tumors, $n=12$; (3) insufficient laboratory data before initial treatment, $\mathrm{n}=39$; (4) known active inflammatory disorders (including autoimmune disease and infection) or active liver or kidney disease, $n=26$. In total, two hundred and thirty-two patients with LSCC were eligible for this study. Written informed consent for the collection of medical data of all patients was obtained. And the ethics committee approved the current study.

All patients were assessed by completed physical examination, fiber laryngoscopy, head and neck MRI (magnetic resonance imaging), abdominal ultrasonography, chest radiography, electrocardiography, and laboratory examination.

\section{Data collection}

Laboratory data collection was performed as the previous studies [18]. Specifically, all laboratory data (blood chemistry analysis) were acquired from patients within 7 days of any surgery. The AGR was calculated using the equation AGR= Albumin/ (total serum protein-albumin); the NLR was calculated using the equation NLR= neutrophils/lymphocytes; the PLR was calculated using the equation PLR= platelets/lymphocytes; the LMR was calculated using the equation $\mathrm{LMR}=$ lymphocytes/monocytes.

\section{Treatment}

The standard treatment of this study was partial or total laryngectomy (+/- neck dissection) and postoperative radio-/chemotherapy according to the National Comprehensive Cancer Network guidelines. Postoperative radiotherapy or chemoradiotherapy was performed in patients with adverse features (including positive margins, pT4 primary, N2 or 3 nodal metastases, extracapsular node/perineural invasion).

\section{Follow-up}

All patients were follow-up for every 3 months in the first 2 years, and every 6 months thereafter for up to 5 years or until death. Follow-up examination including fiber laryngoscopy, neck ultrasonography, MRI. The recurrence was defined as any newly found mass on imaging examination with histologically confirmed by biopsy or surgery. The end follow-up was December 2017.

\section{Statistical analyses}

All analyses were performed using SPSS v22.0 (IBM Corporation, Armonk, New York, USA). Differences among groups were compared by the Chi-square test, Mann-Whitney $U$ test for the different types of variables. Receiver operating characteristic (ROC) curves were plotted to determine the optimal cut-off value for AGR/NLR/PLR/LMR, and the area under the curve (AUC) were compared. The univariate and multivariate analysis were evaluated by the log-rank test and the cox proportional hazard model. A $P<0.05$ was defined as statically significance.

\section{Results}

\section{Clinicopathological Features and Treatment Outcomes}

232 LSCC patients' clinicopathological features as showed in Table 1. Of these patients, $192(82.76 \%)$ were males, $40(17.24 \%)$ were females. Their median age at diagnose was 63 (range, 39-81). According to the $7^{\text {th }}$ edition of the International Union against Cancer/American Joint Committee on Cancer (UICC/AJCC) staging system for LSCC, 113 patients $(48.7 \%)$ were diagnosed stage III or IV. During a $27.3 \pm 18.6$ months' follow-up, 115 patients $(49.6 \%)$ and 78 patients $(33.6 \%)$ were experienced tumor recurrence and death, respectively. The median (range) for AGR, NLR, PLR, LMR was 1.30 (0.75-2.58), $2.98(0.78-20.50), 109.00 \quad(24.00-505.00)$, and 2.56 (0.77-18.87), respectively. And the optimal cut-off value, determined by the ROC analysis for overall survival (OS), for AGR, NLR, PLR, LMR was 1.31 (AUC: $0.707,95 \% \mathrm{CI}: 0.639-0.775, P<0.001$ ), 2.38 (AUC: 0.567, 95\% CI: $0.509-0.641, \quad P=0.044), \quad 116.00$ (AUC:0.607. 95\% CI: $0.535-0.680, P=0.005$ ), and 2.01 (AUC: $0.618, \quad 95 \% \quad \mathrm{CI}: \quad 0.546-0.690, \quad P=0.002$ ), respectively. And the AUC values were statistically compared to evaluate the discrimination ability of every inflammation-based marker (Figure 1). The AGR had significantly higher AUC value than NLR 
$(P=0.011), \operatorname{PLR}(P=0.039)$ and LMR $(P=0.022)$.

\section{Association of preoperative inflammation-based markers with clinical characteristics}

This cohort was divided into 2 groups using the optimal cut-off value for AGR, NLR, PLR and LMR, respectively (Table 2 ). In the AGR subgroups, age, gender, smoking history, drinking history and differentiation grade were found no significant difference among two groups. However, tumor site $(P=0.018)$, larger tumor size $(P<0.001), \mathrm{T}_{3+4}$ stage $(P<0.001)$, lymph node metastasis $(P<0.001)$, and later TNM stage $(\mathrm{III}+\mathrm{IV}, \quad P<0.001)$ were found to be associated with the lower AGR group. In the NLR subgroups, tumor size $(P=0.022), \mathrm{T}_{3+4}$ stage $(P<0.001)$, lymph node metastasis $(P=0.021)$, later TNM stage $(P=0.017)$ were associated with the higher NLR group. In the PLR subgroups, larger tumor size $(P=0.016)$, $\mathrm{T}_{3+4}$ stage $(P=0.011)$, later TNM stage $(P=0.019)$ were associated with higher AGR group significantly. Besides, the lower LMR group was associated with larger tumor size $(P=0.007), \mathrm{T}_{3+4}$ stage $(P<0.001)$, lymph node metastasis $(P<0.001)$, later TNM stage $(P=0.011)$.

Table 1. Baseline characteristics of enrolled patients

\begin{tabular}{lll}
\hline Patient characteristics & $\mathrm{n} / \mathrm{mean} \pm \mathrm{SD}$ \\
\hline Number of patients & 232 \\
Age at diagnosis (yrs, median, range) & $63(39-81)$ \\
$\begin{array}{l}\text { Sex (male/female) } \\
\text { Smokers (\%) }\end{array}$ & $192 / 40$ \\
$\begin{array}{l}\text { Drinkers (\%) } \\
\text { Tumor size (cm) }\end{array}$ & $133(57.7 \%)$ \\
Tumor site (\%) & $91(39.2 \%)$ \\
& & $1.98 \pm 0.94$ \\
& & \\
& Glottic & $152(65.2 \%)$ \\
Differentiation grade & Supraglottic & $68(29.3 \%)$ \\
& Subglottic & $12(5.2 \%)$ \\
T stage III or IV (\%) & Poor & \\
Lymph node metastasis (N+) (\%) & $39(16.8 \%)$ \\
TNM stage III or IV (\%) & & $193(83.2 \%)$ \\
Recurrence (\%) & $106(45.7 \%)$ \\
Death (\%) & $112(48.3 \%)$ \\
Follow-up months (m) & & $113(48.7 \%)$ \\
\hline
\end{tabular}

$\mathbf{A}$

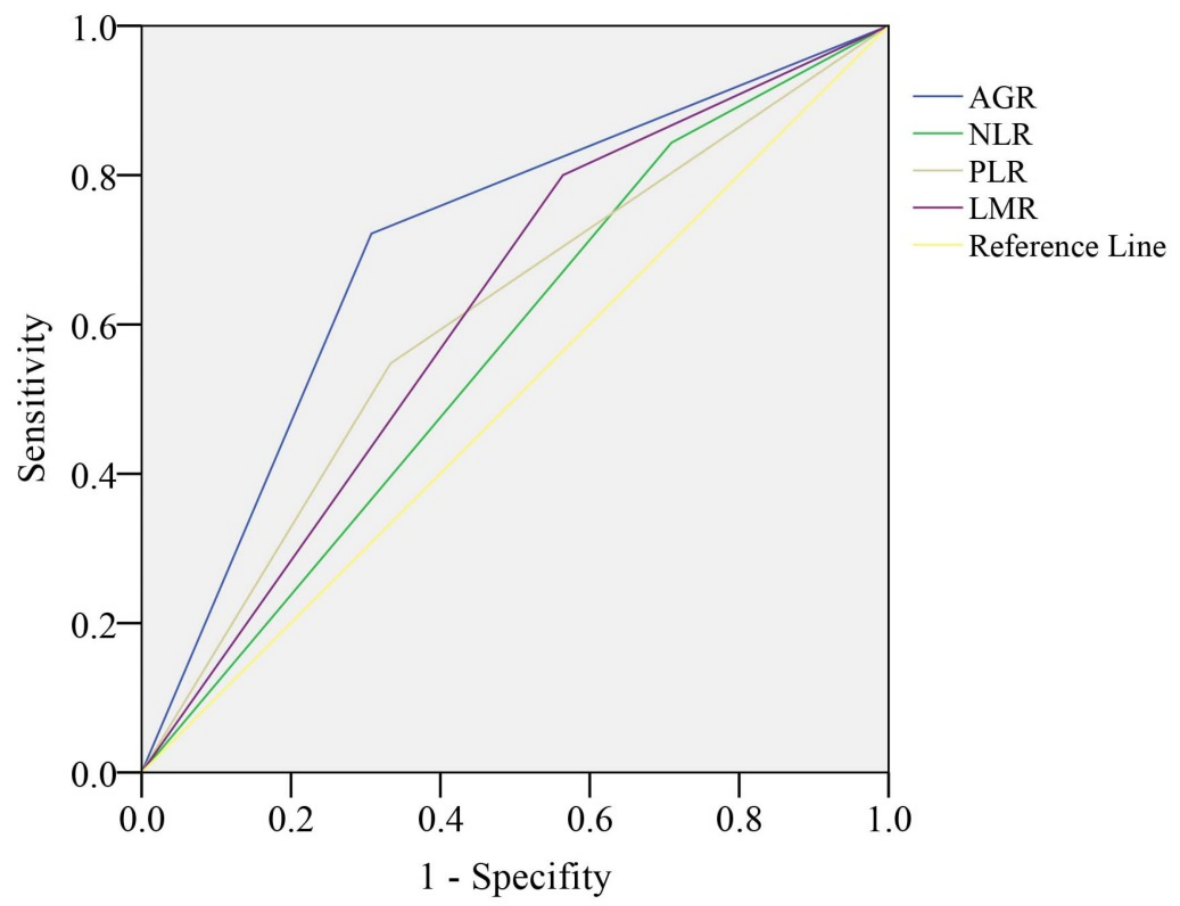

B

\begin{tabular}{ccccc}
\multicolumn{5}{l}{ Comparsion of AUC between inflammation-based markers } \\
\hline Markers & AUC & $95 \%$ CI & $P$ value & $P$ value* \\
\hline AGR & 0.707 & $0.639-0.775$ & $<0.001$ & - \\
NLR & 0.567 & $0.509-0.641$ & 0.044 & 0.011 \\
PLR & 0.607 & $0.535-0.680$ & 0.005 & 0.039 \\
LMR & 0.618 & $0.546-0.690$ & 0.002 & 0.022 \\
\hline AGR: albumin/globulin ratio; NLR: neutrophil/lymphocyte ratio; PLR: platelet/lymphocyte ratio; LMR: lymphocyte/monocyte ratio \\
* Compasion of AUC values between the AGR and other inflammation-based markers was using Z test method
\end{tabular}

Figure 1. (A) ROC curves of the AGR, NLR, PLR, and LMR for survival status among 232 patients with LSCC. (B) Comparison of the area under the ROC curves among the inflammation-based markers for prognosis of LSCC patients. ROC Receiver operating characteristic, AGR albumin/globulin ratio, NLR neutrophil/lymphocyte ratio, PLR platelet/lymphocyte ratio, LMR lymphocyte/monocyte ratio, LSCC laryngeal squamous cell carcinoma. 
Table 2. Correlation between inflammation-based markers and clinicopathological characteristics of LSCC patients

\begin{tabular}{|c|c|c|c|c|c|c|c|c|c|c|c|c|}
\hline Characteristics & AGR & & & NLR & & & PLR & & & LMR & & \\
\hline Age (years) & $<1.31 \mathrm{n}=119$ & $\geq 1.31 \mathrm{n}=113$ & $P$ & $<2.38 \mathrm{n}=48$ & $\geq 2.38 n=184$ & $P$ & $<116 n=130$ & $\geq 116 \mathrm{n}=102$ & $P$ & $<2.01 \mathrm{n}=70$ & $\geq 2.01 \mathrm{n}=162$ & $P$ \\
\hline$<60$ & $54(45.4 \%)$ & $59(52.2 \%)$ & 0.301 & $24(50.0 \%)$ & $89(48.4 \%)$ & 0.832 & $59(43.4 \%)$ & $54(52.9 \%)$ & 0.247 & $36(51.4 \%)$ & $77(47.5 \%)$ & 0.589 \\
\hline$\geq 60$ & $65(54.6 \%)$ & $54(47.8 \%)$ & & $24(50.0 \%)$ & $95(51.6 \%)$ & & $71(54.6 \%)$ & $48(47.1 \%)$ & & $34(48.6 \%)$ & $85(52.5 \%)$ & \\
\hline \multicolumn{13}{|l|}{ Gender } \\
\hline Male & $98(82.4 \%)$ & $94(83.2 \%)$ & 0.859 & $40(83.3 \%)$ & $152(82.6 \%)$ & 0.908 & $107(82.3 \%)$ & $85(83.3 \%)$ & 0.836 & $59(84.3 \%)$ & $133(82.1 \%)$ & 0.681 \\
\hline Female & $21(17.6 \%)$ & $19(16.8 \%)$ & & $8(16.7 \%)$ & $32(17.4 \%)$ & & $23(17.7 \%)$ & $17(16.7 \%)$ & & $11(15.7 \%)$ & $29(17.9 \%)$ & \\
\hline \multicolumn{13}{|l|}{ Smoking history } \\
\hline No & $44(37.0 \%)$ & $55(48.7 \%)$ & 0.156 & $25(52.1 \%)$ & $74(40.2 \%)$ & 0.134 & $58(44.6 \%)$ & $41(40.2 \%)$ & 0.488 & $33(47.1 \%)$ & $66(40.7 \%)$ & 0.821 \\
\hline Yes & $75(63.0 \%)$ & $58(51.3 \%)$ & & $23(47.9 \%)$ & $110(59.8 \%)$ & & $72(55.4 \%)$ & $61(59.8 \%)$ & & $37(52.9 \%)$ & $96(59.3 \%)$ & \\
\hline \multicolumn{13}{|l|}{ Drinking history } \\
\hline No & $70(58.8 \%)$ & $71(63.4 \%)$ & 0.481 & $31(64.6 \%)$ & $110(59.8 \%)$ & 0.542 & $83(63.8 \%)$ & $58(56.9 \%)$ & 0.278 & $36(51.4 \%)$ & $105(64.8 \%)$ & 0.064 \\
\hline Yes & $49(41.2 \%)$ & $41(36.6 \%)$ & & $17(35.4 \%)$ & $74(40.2 \%)$ & & $47(36.2 \%)$ & $44(43.1 \%)$ & & $34(48.6 \%)$ & $57(35.2 \%)$ & \\
\hline \multicolumn{13}{|l|}{ Tumor site } \\
\hline Supraglottic & $46(38.7 \%)$ & $28(24.8 \%)$ & $0.018^{*}$ & $11(22.9 \%)$ & $57(31.0 \%)$ & 0.268 & $37(28.5 \%)$ & $31(30.4 \%)$ & 0.751 & $20(28.6 \%)$ & $48(29.6 \%)$ & 0.874 \\
\hline Glottic\&Subglottic & $73(61.3 \%)$ & $85(75.2 \%)$ & & $37(77.1 \%)$ & $127(69.0 \%)$ & & $93(71.5 \%)$ & $71(69.6 \%)$ & & $50(71.4 \%)$ & $114(70.4 \%)$ & \\
\hline Tumor size & & & $<0.001^{*}$ & & & & & & & & & \\
\hline$\leq 2 \mathrm{~cm}$ & $51(42.9 \%)$ & $69(61.1 \%)$ & & $32(66.7 \%)$ & $88(47.8 \%)$ & $0.022^{*}$ & $76(58.5 \%)$ & $44(43.1 \%)$ & $0.016^{*}$ & $28(40.0 \%)$ & $92(56.8 \%)$ & $0.007^{*}$ \\
\hline$>2 \mathrm{~cm}$ & $68(57.1 \%)$ & $44(38.9 \%)$ & & $16(33.3 \%)$ & $96(52.2 \%)$ & & $54(41.5 \%)$ & $58(56.9 \%)$ & & $42(60.0 \%)$ & $70(43.2 \%)$ & \\
\hline \multicolumn{13}{|l|}{ T Stage } \\
\hline $\mathrm{T} 1+2$ & $58(48.7 \%)$ & $83(73.5 \%)$ & $<0.001^{*}$ & $37(77.1 \%)$ & $104(56.5 \%)$ & $<0.001^{*}$ & $88(67.7 \%)$ & $53(52.0 \%)$ & $0.011^{*}$ & $33(47.1 \%)$ & $108(66.7 \%)$ & $<0.001^{*}$ \\
\hline $\mathrm{T} 3+4$ & $61(51.3 \%)$ & $30(26.5 \%)$ & & $11(22.9 \%)$ & $80(43.5 \%)$ & & $42(32.3 \%)$ & $49(48.0 \%)$ & & $37(52.9 \%)$ & $54(33.3 \%)$ & \\
\hline \multicolumn{3}{|c|}{ Lymph node metastasis } & $<0.001^{*}$ & & & & & & & & & \\
\hline No & $46(38.7 \%)$ & $74(65.5 \%)$ & & $32(66.7 \%)$ & $88(47.8 \%)$ & $0.021^{*}$ & $70(53.8 \%)$ & $50(49.0 \%)$ & 0.471 & $15(21.4 \%)$ & $105(64.8 \%)$ & $<0.001^{*}$ \\
\hline $\mathrm{N}+$ & $73(61.3 \%)$ & $39(34.5 \%)$ & & $16(33.3 \%)$ & $96(52.2 \%)$ & & $60(46.2 \%)$ & $52(51.0 \%)$ & & $55(78.6 \%)$ & $57(35.2 \%)$ & \\
\hline TNM stage & & & $<0.001^{*}$ & & & $0.017^{*}$ & & & $0.019^{*}$ & & & $0.011^{*}$ \\
\hline $\mathrm{I}+\mathrm{II}$ & $42(35.3 \%)$ & $77(68.1 \%)$ & & $32(66.7 \%)$ & $87(47.3 \%)$ & & $75(57.7 \%)$ & $44(43.1 \%)$ & & $27(38.6 \%)$ & $92(56.8 \%)$ & \\
\hline III-IV & $77(64.7 \%)$ & $36(31.9 \%)$ & & $16(33.3 \%)$ & $97(52.7 \%)$ & & $55(42.3 \%)$ & $58(56.9 \%)$ & & $43(61.4 \%)$ & $70(43.2 \%)$ & \\
\hline \multicolumn{2}{|l|}{ Differentiation grade } & & 0.483 & & & 0.402 & & & 0.168 & & & 0.387 \\
\hline Poor & $18(15.1 \%)$ & $21(18.6 \%)$ & & $10(20.8 \%)$ & $29(15.8 \%)$ & & $18(13.8 \%)$ & $21(20.6 \%)$ & & $14(20.0 \%)$ & $25(15.4 \%)$ & \\
\hline Moderate \& Well & $101(84.9 \%)$ & $92(81.4 \%)$ & & $38(79.2 \%)$ & $155(84.2 \%)$ & & $112(86.2 \%)$ & $81(79.4 \%)$ & & $56(80.0 \%)$ & $137(84.6 \%)$ & \\
\hline
\end{tabular}

Abbreviations: HR hazard ratio, 95\% CI 95\% confidencel interval, AGR albumin/globulin ratio

NLR neutrophil/lymphocyte ratio, PLR platelet/lymphocyte ratio, LMR lymphocyte/monocyte ratio, LSCC laryngeal squamous cell carcinoma

${ }^{*} P<0.05$ considered as statistically significant.

\section{Prognostic significance of clinicopathological features in LSCC patients}

In univariate analysis (Table 3), gender, smoking history, drinking history, tumor size showed no significant association with a shorter OS or disease-free survival (DFS). However, tumor site, $\mathrm{T}_{3+4}$ stage, lymph node metastasis, later TNM stage, differentiation grade, AGR, NLR, PLR, and LMR were found to be associated with a shorter OS or DFS. Besides, age $\geq 60$ was associated with OS, not DFS. Multivariate analyses were performed based on those markers with significance in the univariate analysis. We found that lymph node metastasis, later TNM stage, differentiation grade, AGR, NLR, PLR, LMR were independent prognostic markers for OS (Table 4). And, lymph node metastasis, later TNM stage, AGR were still independent prognostic markers for DFS.

\section{Prognostic significance of inflammation-based markers in LSCC patients}

In univariate analysis (Table 3), the shorter OS was significantly associated with AGR (HR: 3.227, 95\% CI: 1.692-4.395, P<0.001), NLR (HR: 1.994, 95\% CI: 1.126-3.374, $P=0.031$ ), PLR (HR: $1.815,95 \%$ CI: 1.160-2.841, $P=0.011$ ), and LMR (HR: 2.291, 95\% CI: 1.344-3.439, $P=0.019)$. And the shorter DFS was significantly associated with AGR (HR: 3.512, 95\% CI: 2.330-5.294, $P<0.001$ ), NLR (HR: 2.295, 95\% CI: 1.312-4.015, $P<0.001$ ), PLR (HR: 1.826, 95\% CI: 1.264-2.638, $P=0.024)$, LMR (HR: 2.283, 95\% CI: 1.433-3.635, $P=0.012$ ).

In multivariate analysis (Table 4), AGR (HR: 3.479, 95\% CI: 2.157-5.612, $P<0.001$ ), NLR (HR: 1.295, 95\% CI: 1.012-3.015, $P=0.044)$, PLR (HR: 1.621, 95\% CI: 1.083-2.427, $P=0.021$ ), LMR (HR: 1.898, 95\% CI: 1.191-2.540, $P=0.017$ ) were independent prognostic markers of the shorter OS. However, NLR, PLR, and LMR showed no significance with the shorter DFS. AGR (HR: 2.595, 95\% CI: 1.477-4.557, P<0.001) remained an independent prognostic marker for the shorter DFS.

Kaplan-Meier survival analysis were performed and survival curves were plotted (Figure 2). Patients with lower AGR had a worse prognosis in 5-year OS ( $44.55 \%$ vs. $75.07 \%, P<0.001)$ and DFS $(26.50 \%$ vs. $71.04 \%, P<0.001)$. The 5-year OS/DFS in patients with higher NLR was worse than lower NLR patients (OS: $77.45 \%$ vs. $55.92 \%, P=0.03$; DFS: $69.92 \%$ vs. $42.73 \%$, $P=0.001)$. Patients with higher PLR had a lower 5-year OS (67.69\% vs. $47.69 \%, P=0.001)$ and DFS (58.38\% vs. $35.34 \%, P=0.001)$. And 5 -year OS $(53.62 \%$ vs. $74.15 \%$, $P=0.01)$ and DFS $(39.62 \%$ vs. $67.93 \%, P<0.001)$ in patients with lower LMR were worse than patients with higher LMR. 
Table 3. Univariate Cox proportional hazards regression analysis for overall survival (OS) and disease-free survival(DFS) in patients with laryngeal squamous cell carcinoma(LSCC)

\begin{tabular}{|c|c|c|c|c|}
\hline & OS & $\mathrm{P}$ & DFS & $\mathrm{P}$ \\
\hline Characteristics & $\mathrm{HR}(95 \% \mathrm{CI})$ & & $\mathrm{HR}(95 \% \mathrm{CI})$ & \\
\hline$\overline{\text { Age(y) }}$ & & $0.022^{*}$ & & 0.432 \\
\hline$<60$ & 1 & & 1 & \\
\hline$\geq 60$ & $1.397(1.111-1.823)$ & & $1.197(0.611-1.323)$ & \\
\hline Gender & & 0.093 & & 0.214 \\
\hline Male & $2.011(0.912-3.447)$ & & $1.921(0.745-2.422)$ & \\
\hline Female & 1 & & 1 & \\
\hline Smoking history & & 0.114 & & 0.192 \\
\hline No & 1 & & 1 & \\
\hline Yes & $2.228(0.891-4.374)$ & & $1.765(0.889-3.018)$ & \\
\hline Drinking history & & 0.427 & & 0.514 \\
\hline No & 1 & & 1 & \\
\hline Yes & $1.772(0.733-2.936)$ & & $1.234(0.533-2.109)$ & \\
\hline Tumor site & & $<0.001^{*}$ & & $<0.001^{*}$ \\
\hline Supraglottic & $2.101(1.506-2.930)$ & & $2.331(1.764-3.081)$ & \\
\hline Glottic\&Subglottic & 1 & & 1 & \\
\hline Tumor size & & 0.068 & & 0.114 \\
\hline$<2 \mathrm{~cm}$ & 1 & & 1 & \\
\hline$\geq 2 \mathrm{~cm}$ & $1.781(0.996-2.578)$ & & $1.334(0.891-2.119)$ & \\
\hline T Stage & & $0.032^{*}$ & & $0.019^{*}$ \\
\hline $\mathrm{T} 1+2$ & 1 & & 1 & \\
\hline $\mathrm{T} 3+4$ & $1.407(1.028-2.542)$ & & $1.604(1.112-2.315)$ & \\
\hline Lymph node metastasis & & $0.024^{*}$ & & $0.033^{*}$ \\
\hline No & 1 & & 1 & \\
\hline $\mathrm{N}+$ & $2.012(1.342-2.997)$ & & $1.387(1.118-2.009)$ & \\
\hline TNM stage & & $<0.001^{*}$ & & $<0.001^{*}$ \\
\hline $\mathrm{I}+\mathrm{II}$ & 1 & & 1 & \\
\hline III-IV & $2.932(1.412-4.976)$ & & $1.668(1.152-2.416)$ & \\
\hline Differentiation grade & & $0.012^{*}$ & & $0.013^{*}$ \\
\hline Poor & $1.667(1.289-2.178)$ & & $1.433(1.198-2.090)$ & \\
\hline Moderate \& Well & 1 & & 1 & \\
\hline AGR & & $<0.001^{*}$ & & $<0.001^{*}$ \\
\hline$<1.31$ & $3.227(1.692-4.395)$ & & $3.512(2.330-5.294)$ & \\
\hline$\geq 1.31$ & 1 & & 1 & \\
\hline NLR & & $0.031^{*}$ & & $<0.001^{*}$ \\
\hline$<2.38$ & 1 & & 1 & \\
\hline$\geq 2.38$ & $1.994(1.126-3.374)$ & & $2.295(1.312-4.015)$ & \\
\hline PLR & & $0.011^{*}$ & & $0.024^{*}$ \\
\hline$<116$ & 1 & & 1 & \\
\hline$\geq 116$ & $1.815(1.160-2.841)$ & & $1.826(1.264-2.638)$ & \\
\hline LMR & & $0.019^{*}$ & & $0.012^{*}$ \\
\hline$<2.01$ & $2.291(1.344-3.439)$ & & $2.283(1.433-3.635)$ & \\
\hline$\geq 2.01$ & 1 & & 1 & \\
\hline \multicolumn{4}{|c|}{$\begin{array}{l}\text { Abbreviations: } H R \text { hazard ratio, } 95 \% \text { CI 95\% confidencel interval, } A G R \\
\text { albumin/globulin ratio }\end{array}$} & \\
\hline
\end{tabular}

\section{Discussion}

AGR, along with other inflammation-based markers, in the current study were associated with aggressive clinicopathological features and poor prognosis in LSCC patients. And AGR had a significantly higher AUC value compared with other inflammation-based markers in terms of predicting the prognosis of LSCC patients. To the best of our knowledge, this is the first study to specifically focus on the comparison of the prognostic value of AGR with other inflammation-based markers in cancer patients.

Over the last decade, various inflammationbased markers have been reported their prognostic role in many cancers [19-23], including LSCC [13-18]. In our previous report, we firstly described AGR as a prognostic marker for patients with LSCC. However, none of these studies have compared their prognostic value. In the current study, we found that all inflammation-based markers (NLR, PLR, LMR, AGR) associated with aggressive clinicopathological features (such as tumor size, lymph node metastasis, and late TNM stage) and have a significantly prognostic value for patients with LSCC, which was consistent with other reports.

Table 4. Multivariate Cox proportional hazards regression analysis for overall survival (OS) and disease-free survival (DFS) in patients with laryngeal squamous cell carcinoma (LSCC)

\begin{tabular}{|c|c|c|c|c|}
\hline & OS & $P$ & DFS & $P$ \\
\hline Characteristics & $\mathrm{HR}(95 \% \mathrm{CI})$ & & $\mathrm{HR}(95 \% \mathrm{CI})$ & \\
\hline Age(y) & & 0.757 & & - \\
\hline$<60$ & 1 & & - & \\
\hline$\geq 60$ & $1.127(0.781-1.423)$ & & & \\
\hline Tumor site & & 0.379 & & 0.543 \\
\hline Supraglottic & $1.262(0.634-2.513)$ & & $2.026(0.564-2.328)$ & \\
\hline Glottic\&Subglottic & 1 & & 1 & \\
\hline T Stage & & 0.393 & & 0.229 \\
\hline $\mathrm{T} 1+2$ & 1 & & 1 & \\
\hline $\mathrm{T} 3+4$ & $1.007(0.450-2.004)$ & & $1.219(0.512-1.925)$ & \\
\hline Lymph node metastasis & & $0.021^{*}$ & & $0.048^{*}$ \\
\hline N0 & 1 & & 1 & \\
\hline $\mathrm{N}+$ & $1.512(1.332-2.294)$ & & $1.561(1.322-2.976)$ & \\
\hline TNM stage & & $<0.001^{*}$ & & $<0.001^{*}$ \\
\hline $\mathrm{I}+\mathrm{II}$ & 1 & & 1 & \\
\hline III-IV & $2.014(1.397-2.904)$ & & $2.403(1.768-3.266)$ & \\
\hline Differentiation grade & & $0.041^{*}$ & & 0.376 \\
\hline Poor & $1.327(1.008-2.178)$ & & $1.234(0.582-1.992)$ & \\
\hline Moderate \& Well & 1 & & 1 & \\
\hline AGR & & $<0.001^{*}$ & & $<0.001^{*}$ \\
\hline$<1.31$ & $3.479(2.157-5.612)$ & & $2.595(1.477-4.557)$ & \\
\hline$\geq 1.31$ & 1 & & 1 & \\
\hline NLR & & $0.044^{*}$ & & 0.611 \\
\hline$<2.38$ & 1 & & 1 & \\
\hline$\geq 2.38$ & $1.295(1.012-3.015)$ & & $1.243(0.536-2.883)$ & \\
\hline PLR & & $0.021^{*}$ & & 0.089 \\
\hline$<116$ & 1 & & 1 & \\
\hline$\geq 116$ & $1.621(1.083-2.427)$ & & $1.546(0.946-2.527)$ & \\
\hline LMR & & $0.017^{*}$ & & 0.874 \\
\hline$<2.01$ & $1.898(1.191-2.540)$ & & $1.104(0.446-1.950)$ & \\
\hline$\geq 2.01$ & 1 & & 1 & \\
\hline \multicolumn{5}{|c|}{$\begin{array}{l}\text { Abbreviations: HR hazard ratio, 95\% CI 95\% confidence interval, AGR } \\
\text { albumin/globulin ratio }\end{array}$} \\
\hline $\begin{array}{l}\text { NLR neutrophil/lymph } \\
\text { lymphocyte/monocyte }\end{array}$ & ratio, $P L R$ platele & t/lymph & hocyte ratio, $L M R$ & \\
\hline
\end{tabular}

It is widely recognized that cancer-associated inflammation plays a significant role in tumor progression [5, 24], though the exact mechanism is still unclear. At an early stage of cancer progression, various cytokines generated by cancer cells could recruit inflammatory cells that creating microenvironment, facilitating tumor growth, geomantic instabilities, and angiogenesis [25-27]. Neutrophils could promote cancer cells metastasis by secreting circulating growth factors [28]. Furthermore, neutrophils could weaken T lymphocytes' function 
$\mathbf{A}$

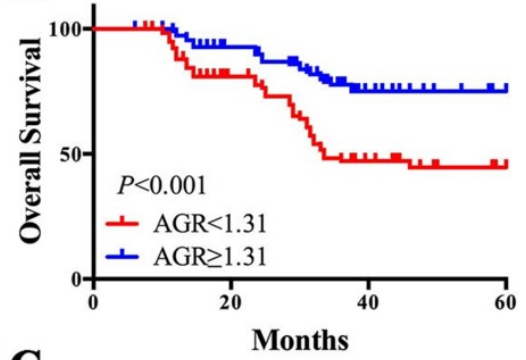

C

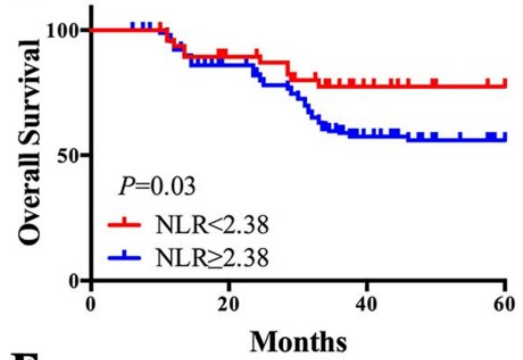

$\mathbf{E}$

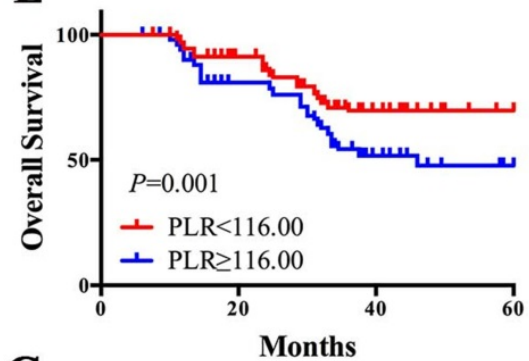

G

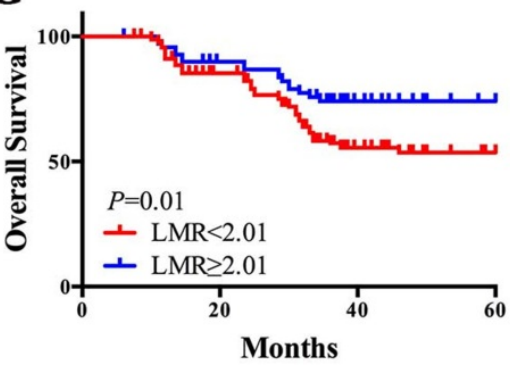

B

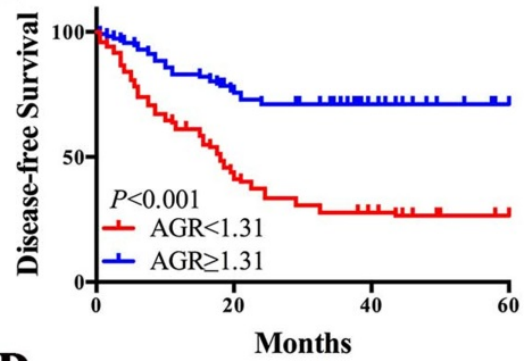

D

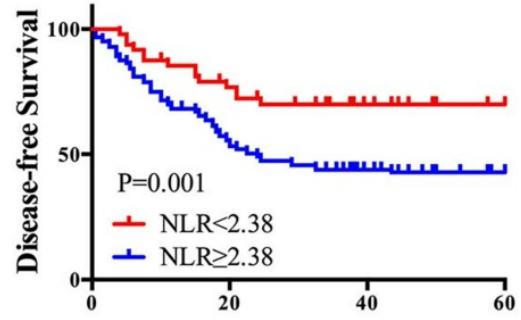

F

Months

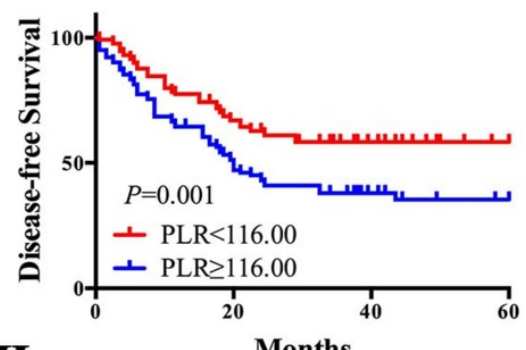

H

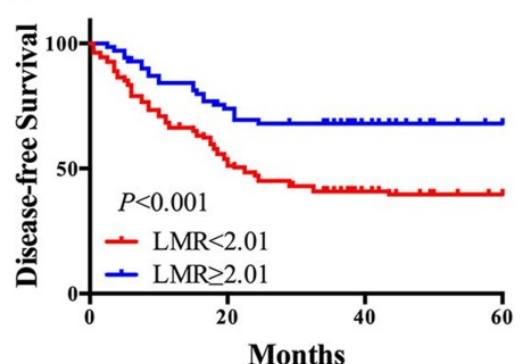

Figure 2. Pretreatment inflammation-based markers and prognosis of $L S C C$ patients. $A G R<1.31$ was associated with poor OS (A) and DFS (B); NLR $\geq 2.38$ was associated with poor OS (C) and DFS (D); PLR $\geq 116$ was associated with poor OS (E) and DFS (F); LMR<2.01 was associated with poor OS (G) and DFS (H). AGR albumin/globulin ratio, NLR neutrophil/lymphocyte ratio, PLR platelet/lymphocyte ratio, LMR lymphocyte/monocyte ratio, LSCC laryngeal squamous cell carcinoma.

and promote cancer progression [29-31]. Lymphocytes induced cell death and inhibiting tumor cell migration and proliferation. Studies demonstrated that better prognosis was associated with a higher proportion of lymphocytes infiltration into the tumor stroma [32, 33]. In addition, platelets have been reported to directly interact with tumor cells by activating TGF- $\beta /$ Smad and NF-kB pathways, inducing cancer cells epithelial-mesenchymal transition and thus promote cancer metastasis [34]. Furthermore, monocytes have been reported to promote tumor metastasis by circulation and the tumor-monocytes-endothelial interaction $[35,36]$. Therefore, NLR, PLR, LMR play an important role in the prognosis of LSCC.
AGR not only reflects the systemic inflammatory responses, but dystrophia. Firstly, albumin has been widely used to evaluate the nutritional status and to predict the prognosis of cancer patients. Albumin has been reported various anticancer capabilities, including steadying cell growth and managing DNA replication, buffering many biochemical alterations, and its antioxidant effects which may against carcinogens [37]. Secondly, malnutrition and inflammation could prevent the synthesis of albumin. For instance, interleukin- 6 promotes the generation of acute-phase reaction proteins in the liver and regulation of the synthesis of albumin by liver cells, whereas tumor necrosis factor can down-regulate the albumin gene transcription and increase the 
permeability of the microvasculature which leading to increased transcapillary passage of albumin [38]. Thirdly, high levels of globulins caused by aggregation of the acute-phase proteins and immunoglobulins, which reflect an inflammatory state in the tumor microenvironment [38].

In addition, we compared the prognosis value of the NLR, PLR, LMR and AGR by $Z$ test in ROC analysis. This methodology has been validated in previous studies [39]. The results have shown that the AUC value of AGR is significantly more than the NLR, PLR, and LMR, which indicated that AGR may have a better discriminatory ability than other inflammatory markers in terms of prognosis for patients with LSCC. Since authors believe that not only systemic inflammation but nutritional status plays a role in cancer progression [40-42]. The AGR is the combination of these two predictors of adverse outcomes. Thus, it may explain AGR have a better predictive value in LSCC patients.

There are several limitations to the current study. The retrospective nature of this study may lead to an inevitable bias; therefore, an independent cohort with long-term follow-up is needed to further analyze the predictive value of these factors in LSCC patients. Additionally, the AGR were assessed at the preoperative single time point. The changes in blood over time and in response to treatment, and their relationships to the prognosis of LSCC patients may be the subject in the future study.

\section{Conclusion}

Our findings show that the AGR may serve as a promising prognostic factor in LSCC patients, and have a better discriminatory ability than other inflammation-based markers. Further studies are warranted to validate the predictive role of AGR in a larger, prospective, multi-centers cohort.

\section{Acknowledgements}

This work received a grant from the National Natural Science Foundation of China (No. 81760320).

\section{Competing Interests}

The authors have declared that no competing interest exists.

\section{References}

1. Siegel RL, Miller KD, Jemal A. Cancer statistics, 2018. CA Cancer J Clin. 2018; 68: 7-30.

2. Chen W, Zheng R, Baade PD, Zhang S, Zeng H, Bray F, et al. Cancer statistics in China, 2015. CA Cancer J Clin. 2016; 66: 115-32.

3. Trinchieri G. Cancer and inflammation: an old intuition with rapidly evolving new concepts. Annu Rev Immunol. 2012; 30: 677-706.

4. Diakos CI, Charles KA, McMillan DC, Clarke SJ. Cancer-related inflammation and treatment effectiveness. Lancet Oncol. 2014; 15: e493-503.

5. Mantovani A. Cancer: Inflaming metastasis. Nature. 2009; 457: 36-7.
6. Iyengar NM, Gucalp A, Dannenberg AJ, Hudis CA. Obesity and Cancer Mechanisms: Tumor Microenvironment and Inflammation. J Clin Oncol. 2016; 34: 4270-6.

7. Kusumanto YH, Dam WA, Hospers GA, Meijer C, Mulder NH. Platelets and granulocytes, in particular the neutrophils, form important compartments for circulating vascular endothelial growth factor. Angiogenesis. 2003; 6: 283-7.

8. Teramukai S, Kitano T, Kishida Y, Kawahara M, Kubota K, Komuta K, et al. Pretreatment neutrophil count as an independent prognostic factor in advanced non-small-cell lung cancer: an analysis of Japan Multinational Trial Organisation LC00-03. Eur J Cancer. 2009; 45: 1950-8.

9. Lanca T, Silva-Santos B. The split nature of tumor-infiltrating leukocytes: Implications for cancer surveillance and immunotherapy. Oncoimmunology. 2012; 1: 717-25.

10. Palumbo JS, Talmage KE, Massari JV, La Jeunesse CM, Flick MJ, Kombrinck $\mathrm{KW}$, et al. Platelets and fibrin(ogen) increase metastatic potential by impeding natural killer cell-mediated elimination of tumor cells. Blood. 2005; 105: 178-85.

11. Klinger $\mathrm{MH}$, Jelkmann W. Role of blood platelets in infection and inflammation. J Interferon Cytokine Res. 2002; 22: 913-22.

12. Rosenberg SA. Progress in human tumour immunology and immunotherapy. Nature. 2001; 411: 380-4.

13. Kara M, Uysal S, Altinisik U, Cevizci S, Guclu O, Derekoy FS. The pre-treatment neutrophil-to-lymphocyte ratio, platelet-to-lymphocyte ratio, and red cell distribution width predict prognosis in patients with laryngeal carcinoma. Eur Arch Otorhinolaryngol. 2017; 274: 535-42.

14. Tu XP, Qiu QH, Chen LS, Luo XN, Lu ZM, Zhang SY, et al. Preoperative neutrophil-to-lymphocyte ratio is an independent prognostic marker in patients with laryngeal squamous cell carcinoma. BMC Cancer. 2015; 15: 743.

15. Wang J, Wang S, Song X, Zeng W, Wang S, Chen F, et al. The prognostic value of systemic and local inflammation in patients with laryngeal squamous cell carcinoma. Onco Targets Ther. 2016; 9: 7177-85.

16. Yu ST, Zhou Z, Cai O, Liang F, Han P, Chen R, et al. Prognostic value of the C-reactive protein/albumin ratio in patients with laryngeal squamous cell carcinoma. Onco Targets Ther. 2017; 10: 879-84.

17. Wong BY, Stafford ND, Green VL, Greenman J. Prognostic value of the neutrophil-to-lymphocyte ratio in patients with laryngeal squamous cell carcinoma. Head Neck. 2016; 38 Suppl 1: E1903-8.

18. Chen WZ, Yu ST, Xie R, Lv YX, Xu DB, Yu JC. Preoperative albumin/globulin ratio has predictive value for patients with laryngeal squamous cell carcinoma. Oncotarget. 2017; 8: 48240-7.

19. Cannon NA, Meyer J, Iyengar P, Ahn C, Westover KD, Choy H, et al. Neutrophil-lymphocyte and platelet-lymphocyte ratios as prognostic factors after stereotactic radiation therapy for early-stage non-small-cell lung cancer. J Thorac Oncol. 2015; 10: 280-5.

20. Wang SC, Chou JF, Strong VE, Brennan MF, Capanu M, Coit DG. Pretreatment Neutrophil to Lymphocyte Ratio Independently Predicts Disease-specific Survival in Resectable Gastroesophageal Junction and Gastric Adenocarcinoma. Ann Surg. 2016; 263: 292-7.

21. Chan JC, Chan DL, Diakos CI, Engel A, Pavlakis N, Gill A, et al. The Lymphocyte-to-Monocyte Ratio is a Superior Predictor of Overall Survival in Comparison to Established Biomarkers of Resectable Colorectal Cancer. Ann Surg. 2017; 265: 539-46.

22. Saeed L, Patnaik MM, Begna KH, Al-Kali A, Litzow MR, Hanson CA, et al. Prognostic relevance of lymphocytopenia, monocytopenia and lymphocyte-to-monocyte ratio in primary myelodysplastic syndromes: a single center experience in 889 patients. Blood Cancer J. 2017; 7: e550.

23. Chan JCY, Diakos CI, Chan DLH, Engel A, Pavlakis N, Gill A, et al. A Longitudinal Investigation of Inflammatory Markers in Colorectal Cancer Patients Perioperatively Demonstrates Benefit in Serial Remeasurement. Ann Surg. 2018; 267: 1119-25.

24. Deng T, Lyon CJ, Bergin S, Caligiuri MA, Hsueh WA. Obesity, Inflammation, and Cancer. Annu Rev Pathol. 2016; 11: 421-49.

25. Elinav $\mathrm{E}$, Nowarski $\mathrm{R}$, Thaiss $\mathrm{CA}, \mathrm{Hu}$ B, Jin $\mathrm{C}$, Flavell RA. Inflammation-induced cancer: crosstalk between tumours, immune cells and microorganisms. Nat Rev Cancer. 2013; 13: 759-71.

26. Ino $\mathrm{Y}$, Yamazaki-Itoh R, Shimada $\mathrm{K}$, Iwasaki M, Kosuge $\mathrm{T}$, Kanai $\mathrm{Y}$, et al. Immune cell infiltration as an indicator of the immune microenvironment of pancreatic cancer. Br J Cancer. 2013; 108: 914-23.

27. Roxburgh CS, McMillan DC. Cancer and systemic inflammation: treat the tumour and treat the host. Br J Cancer. 2014; 110: 1409-12.

28. Fondevila C, Metges JP, Fuster J, Grau JJ, Palacin A, Castells A, et al. p53 and VEGF expression are independent predictors of tumour recurrence and survival following curative resection of gastric cancer. Br J Cancer. 2004; 90 : 206-15.

29. Pillay J, Kamp VM, van Hoffen E, Visser T, Tak T, Lammers JW, et al. A subset of neutrophils in human systemic inflammation inhibits $\mathrm{T}$ cell responses through Mac-1. J Clin Invest. 2012; 122: 327-36.

30. Sabbione F, Gabelloni ML, Ernst G, Gori MS, Salamone G, Oleastro M, et al. Neutrophils suppress gammadelta T-cell function. Eur J Immunol. 2014; 44: 819-30.

31. Fridlender ZG, Albelda SM, Granot Z. Promoting metastasis: neutrophils and T cells join forces. Cell Res. 2015; 25: 765-6.

32. Jung AC, Guihard S, Krugell S, Ledrappier S, Brochot A, Dalstein V, et al. CD8-alpha T-cell infiltration in human papillomavirus-related oropharyngeal carcinoma correlates with improved patient prognosis. Int J Cancer. 2013; 132: E26-36. 
33. Oguejiofor K, Hall J, Slater C, Betts G, Hall G, Slevin N, et al. Stromal infiltration of CD8 T cells is associated with improved clinical outcome in HPV-positive oropharyngeal squamous carcinoma. Br J Cancer. 2015; 113: 886-93.

34. Labelle M, Begum S, Hynes RO. Direct signaling between platelets and cancer cells induces an epithelial-mesenchymal-like transition and promotes metastasis. Cancer Cell. 2011; 20: 576-90.

35. Evani SJ, Prabhu RG, Gnanaruban V, Finol EA, Ramasubramanian AK. Monocytes mediate metastatic breast tumor cell adhesion to endothelium under flow. FASEB J. 2013; 27: 3017-29.

36. Rukoyatkina N, Butt E, Subramanian H, Nikolaev VO, Mindukshev I, Walter $\mathrm{U}$, et al. Protein kinase A activation by the anti-cancer drugs ABT-737 and thymoquinone is caspase-3-dependent and correlates with platelet inhibition and apoptosis. Cell Death Dis. 2017; 8: e2898.

37. Seaton K. Albumin concentration controls cancer. J Natl Med Assoc. 2001; 93: $490-3$.

38. Gabay C, Kushner I. Acute-phase proteins and other systemic responses to inflammation. N Engl J Med. 1999; 340: 448-54.

39. Camp RL, Dolled-Filhart M, Rimm DL. X-tile: a new bio-informatics tool for biomarker assessment and outcome-based cut-point optimization. Clin Cancer Res. 2004; 10: 7252-9.

40. Cheng Y, Li H, Deng Y, Tai Y, Zeng K, Zhang Y et al. Cancer-associated fibroblasts induce PDL1+ neutrophils through the IL6-STAT3 pathway that foster immune suppression in hepatocellular carcinoma. Cell Death Dis. 2018;9(4):422.

41. Mao FY, Zhao YL, Lv YP, Teng YS, Kong H, Liu YG, et al. CD45+CD33lowCD11bdim myeloid-derived suppressor cells suppress CD8+ $\mathrm{T}$ cell activity via the IL-6/IL-8-arginase I axis in human gastric cancer. Cell Death Dis. 2018;9(7):763.

42. Marwick JA, Mills R, Kay O, Michail K, Stephen J, Rossi AG, et al. Neutrophils induce macrophage anti-inflammatory reprogramming by suppressing NF-KB activation. Cell Death Dis. 2018;9(6):665. 